\title{
Assessment for sonographic grading scales of the body fat changes related to the lipoatrophic findings of HIV patients in treatment with HAART
}

\author{
R Gulizia*1, C Gervasoni ${ }^{2}, \mathrm{M} \mathrm{Ortu}^{2}, \mathrm{M}$ Galli$^{2}$ and C Filice ${ }^{1}$
}

\author{
Address: ${ }^{1}$ Infectious and Tropical Disease Division of Foundation IRCCS San Matteo, pavia, Italy and ${ }^{2} \mathrm{III}^{\circ}$ Division of Infectious and Tropical \\ Disease, L.Sacco Hospital, Milan, Italy \\ * Corresponding author
}

\author{
from Ninth International Congress on Drug Therapy in HIV Infection \\ Glasgow, UK. 9-13 November 2008 \\ Published: 10 November 2008 \\ Journal of the International AIDS Society 2008, I I (SuppI I):PII $\quad$ doi:I0.II86/I758-2652-II-SI-PII9
}

This abstract is available from: http://www.jiasociety.org/content/II/SI/PII 9

(c) 2008 Gulizia et al; licensee BioMed Central Ltd.

\section{Purpose of the study}

To assess the accuracy of a sonographic based grading scale (US-GS) in identification of body fat changes (BFCs) related to lipoatrophy (LA) in HIV patients treated with antiretrovirals, compared with the HIV Outpatient Study (HOPS) based clinical diagnoses.

\section{Methods}

We enrolled non-obese HIV patients (73 males and 42 females; body mass index $<27 \mathrm{~kg} / \mathrm{m} 2$ ). Clinical assessments of BFCs were defined by correspondence between the self-reports of patients and medical examinations. Fat redistribution was scored separately for face, arm and lower limbs on a scale ranging from 0 (absence) to 1 (mid) and 2-3 (severe BFCs). US scans of subcutaneous fat thickness (SFT) were performed at 3 reference points: transversal scan at the deepest point of Bichat pad (facial SFT), long dorsal scan of arm (brachial SFT), and long scan of anterior face of thigh (crural SFT).

\section{Summary of results}

There were no significant differences in demographics (age, body mass index) or clinical features (CD4 T lymphocytes, duration of HIV infection and duration of HAART) between males or females with no LA (HOPS grading scale 0 ), or those with mid LA (HOPS grading scale 1) or severe LA (HOPS grading scale 2-3). Significant differences were found in US assessments for each corresponding HOPS-GS ( $p$ ranged from $<0.03$ to
0.0004). Diagnostic thresholds were identified for the assessment of each degree of LA severity (US-GS 0 vs. USGS 1 and US-GS 2-3) for facial, brachial and crural LA, with related sensitivity (range: 70-99\%), specificity (range: 70-95\%), positive likelihood ratios (range: 2.8311.2 ) and negative likelihood ratios (range: 0.07-0.41). A logistic regression showed that the US-GS correctly diagnosed the $80.9 \%$ of all cases of LA (regression coefficient: 2.1 (SE: 0.54$), \mathrm{p}<0.0001)$ with an odds ratio of $8.1(95 \%$ CI: 2.8 to 23.5$)$.

\section{Conclusion}

US shows significant correlation with the HOPS based assessments. Significant areas under the ROC curves, distinct diagnostic thresholds with high sensitivity, specificity and likelihood ratios suggest that US is reliable for the diagnosis of LA. Compared to the HOPS, the US-GS shows high percentages of correctly classified cases of LA. Serial assessments could identify early BFCs related to HAART or be used to prevent advanced stages. It could suggest the need for proactive switching therapies or identify the SFT recovery in response to reactive switching therapies. 